\title{
High power sub-200fs pulse generation from a colliding pulse modelocked VECSEL
}

Alexandre Laurain

Declan Marah

Robert Rockmore

John G. Mclnerney

Jorg Hader

Antje Ruiz Perez

Stephan W. Koch

Wolfgang Stolz

Jerome V. Moloney 


\title{
High power sub-200fs pulse generation from a colliding pulse modelocked VECSEL
}

\author{
Alexandre Laurain ${ }^{a}$, Declan Marah $^{b}$, Robert Rockmore ${ }^{a}$, John G. McInerney ${ }^{b}$, Jorg Hader ${ }^{a}$, \\ Antje Ruiz Perez ${ }^{c}$, Stephan W. Koch ${ }^{a, c}$, Wolfgang Stolz ${ }^{c}$, and Jerome V. Moloney ${ }^{a}$. \\ ${ }^{a}$ College of Optical Sciences, University of Arizona, Tucson, Arizona 85721, USA; \\ ${ }^{b}$ Department of Physics and Tyndall Institute, University College, Cork, Ireland. \\ ${ }^{c}$ Physics and Material Sciences Center, Philipps Universität, Marburg 35032, Germany.
}

\begin{abstract}
We present a passive and robust mode-locking scheme for a Vertical External Cavity Surface Emitting Laser (VECSEL). We placed the semiconductor gain medium and the semiconductor saturable absorber mirror (SESAM) strategically in a ring cavity to provide a stable colliding pulse operation. With this cavity geometry, the two counter propagating pulses synchronize on the SESAM to saturate the absorber together. This minimizes the energy lost and creates a transient carrier grating due to the interference of the two beams. The interaction of the two counter-propagating pulses in the SESAM is shown to extend the range of the modelocking regime and to enable higher output power when compared to the conventional VECSEL cavity geometry. In this configuration, we demonstrate a pulse duration of $195 \mathrm{fs}$ with an average power of $225 \mathrm{~mW}$ per output beam at a repetition rate of $2.2 \mathrm{GHz}$, giving a peak power of $460 \mathrm{~W}$ per beam. The remarkable robustness of the modelocking regime is discussed and a rigorous pulse characterization is presented.
\end{abstract}

Keywords: VECSEL, OPSL, semiconductor, modelocking, Colliding pulses, ultra-short pulses.

\section{INTRODUCTION}

The passive mode locking of vertical cavity surface emitting lasers (VECSELs) is a very attractive technique to generate ultrashort pulses at high repetition rates. First, the high finesse of the external optical cavity can provide a high power circular beam with a very low divergence, close to the diffraction limit. ${ }^{1}$ A high beam quality is particularly important for non linear applications, such as supercontinuum or frequency comb generation $^{2}$ where an efficient coupling into a non linear medium (single mode fiber, semiconductor waveguide, etc) is necessary. It is also very important for applications requiring high power density such as multi-photon imaging, ${ }^{3}$ or material processing. ${ }^{4}$ Secondly, the semiconductor gain medium of the VECSEL is advantageous for applications requiring high repetition rates $(>1 \mathrm{GHz})$, as the relatively short carrier lifetime largely suppresses spontaneous Q-switching instabilities, often observed with dielectric gain media. ${ }^{5}$ The passive modelocking of VECSELs has been demonstrated with semiconductor saturable absorber mirror (SESAM) at repetition rates ranging from $85 \mathrm{MHz}$ to $100 \mathrm{GHz},{ }^{6,7}$ and at operating wavelength ranging from $665 \mathrm{~nm}^{8}$ to $1960 \mathrm{~nm} .{ }^{9}$ To date, the best performance in terms of power and pulse duration has been demonstrated between 960 and $1080 \mathrm{~nm},{ }^{10,11}$ where the semiconductor media benefit from the maturity and advantages of GaAs-based materials.

The output power with ultra-short pulses is often limited by the stability of the modelocking regime. A high pumping level often leads to harmonic mode-locking when the repetition rate is sufficiently low, and/or to side pulses when the repetition rate is faster than the carrier lifetime $(>1 G H z)$. A recent theoretical study revealed that in the high excitation regime, a pulse duration in the order of $100 \mathrm{fs}$ can bleach out the non-equilibrium carrier distributions to promote independent carrier reservoirs leading to the emergence of multiple pulse waveforms, ${ }^{12}$ ultimately leading to a trade-off between the pulse duration and the average power achievable.

Here we present a robust modelocking technique where the gain chip and the SESAM are placed in a ring cavity to create colliding pulses in the absorber, reducing the effective losses of the absorber while providing a

Further author information: (Send correspondence to A. Laurain)

A. Laurain: E-mail: alaurain@optics.arizona.edu

Vertical External Cavity Surface Emitting Lasers (VECSELs) VII, edited by Michael Jetter, Proc. of SPIE

Vol. 10087, 100870E · @ 2017 SPIE · CCC code: 0277-786X/17/\$18 · doi: 10.1117/12.2252525 
symmetric gain for the two counter-propagating pulses. ${ }^{13}$ First, we describe in detail the cavity geometry and the design of the semiconductor structure used. Then we present the experimental results with a throughout pulse characterization.

\section{COLLIDING PULSE VECSEL DESIGN AND SETUP}

In a ring laser cavity two beams can coexist and propagate in either directions and interact with each other inside the cavity, provided that they cross in a medium with a non zero susceptibility. If the absorption of the saturable absorber is slightly modulated by the fluctuation of the intracavity power, a pulse-shaping mechanism may occur and provide a modelocked state where two counter-propagating pulses automatically synchronize their flight in the cavity to cross in the SESAM and minimize the losses.

If the counter propagating beams are spatially coherent and share the same polarization, the superposition of the two pulses produces interferences which causes a transverse transient carrier grating in the SESAM. When the pulses are perfectly synchronized, the field intensity on the SESAM has a maximum 4 times higher than the intensity of one beam alone, resulting in a smaller saturation fluence. ${ }^{13}$ The field intensity distribution of two synchronized beam colliding with an angle of $7^{\circ}$ is represented on Fig. 1.

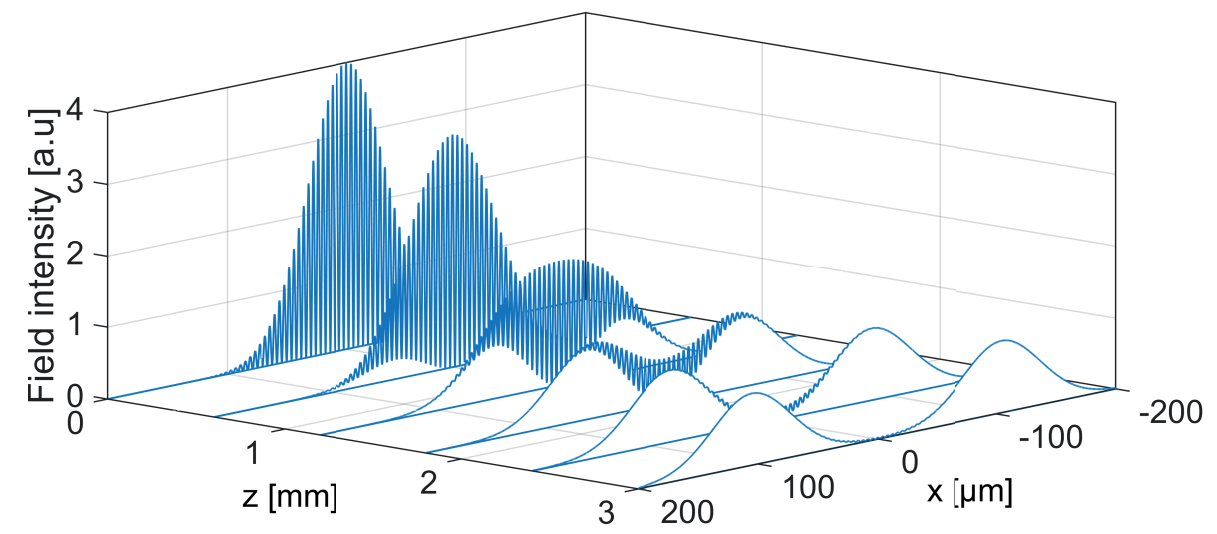

Figure 1. Field intensity distribution of two gaussian beams colliding in a SESAM with an angle of $7^{\circ}$ at $z=0$.

Thanks to the higher field intensity provided by the interference of the pulses, a smaller mode area ratio between the VECSEL gain and the SESAM can be used to provide stable modelocking. We can thus increase the mode size on the absorber to reduce the thermal impedance and increase the damage threshold. Another advantage of such cavity configuration is the reduced group delay dispersion (GDD) of the cavity since the pulses hit the gain chip only once per round trip and the gain chip is often the main non-linear contributor to the GDD. In a more standard V-shaped cavity, where the chip is placed as a folding mirror, the contribution of the chip to the GDD is doubled. We should note however that a single pass on the chip also reduces the modal gain, requiring a smaller output coupler transmission.

\subsection{VECSEL structure design}

The design of the VECSEL structure investigated is illustrated on Fig 2. It is designed for an emission wavelength centered at $990 \mathrm{~nm}$. It is grown by MOCVD on a GaAs substrate. It contains two consecutive semiconductor Bragg mirrors consisting of 23 and 13 pairs of AlAs/AlGaAs quarter-wave layers, transparent at the pump wavelength of $790 \mathrm{~nm}$, to reflect both the lasing and the pump wavelength respectively. It is followed by the active region, an InGaP cap layer and a single layer of $\mathrm{Si}_{3} \mathrm{~N}_{4}$ anti-reflection coating. The active region contains 8 InGaAs QWs, placed in pairs on 4 antinodes of the field. The structure is grown as a bottom emitter and is bonded to diamond following the procedure described here ${ }^{1}$ for optimal thermal management. The dielectric coating is optimized to provide a low group delay dispersion (GDD) and broadband gain.

The SESAM used was grown by MBE on a GaAs substrate. It consists of 24 pairs of AlAs/GaAs followed by a single InGaAs QW placed in close proximity to the surface to provide a high carrier recombination velocity 


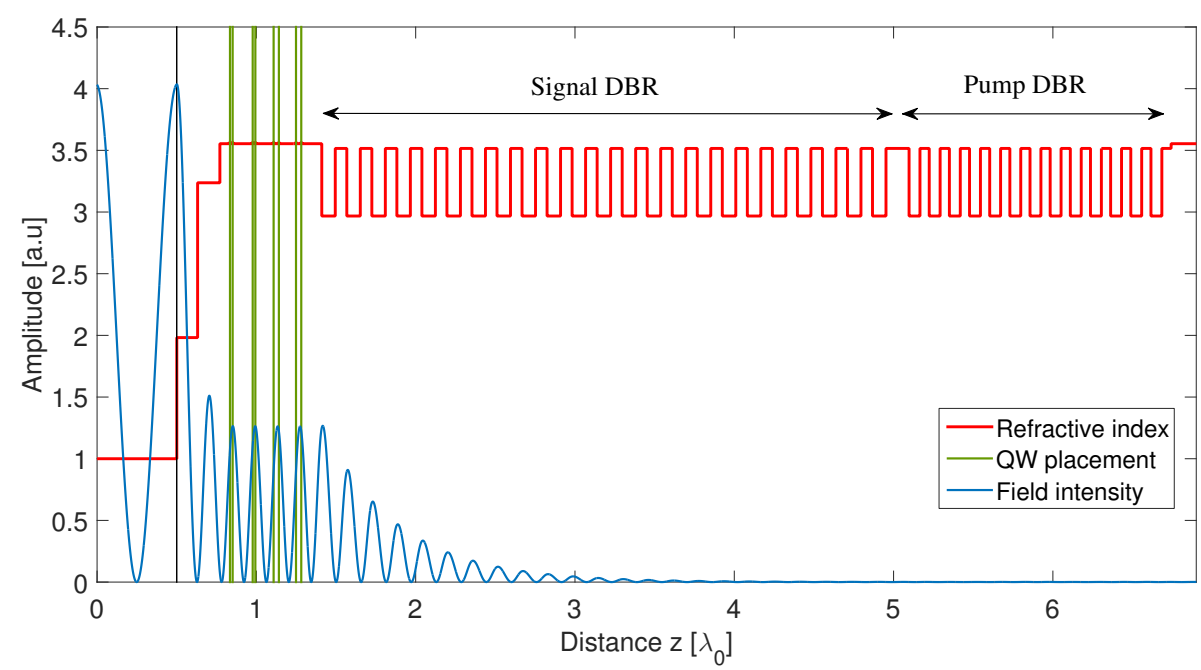

Figure 2. Design of the full VECSEL structure. The field intensity was calculated for an angle of incidence of $7^{\circ}$. The thickness is normalized to the nominal wavelength of $990 \mathrm{~nm}$.

via tunneling to surface states. The surface was also coated with a $\mathrm{Si}_{3} \mathrm{~N}_{4}$ layer for dispersion management. The GDD of the gain structure and SESAM measured at room temperature and normal incidence is shown on Fig. 3.

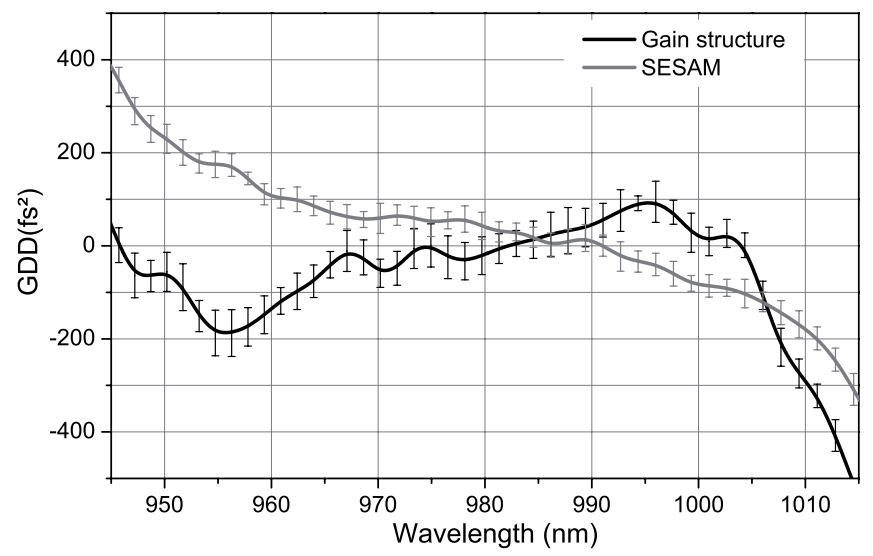

Figure 3. Measured group delay dispersion spectrum of the SESAM and VECSEL gain structure. The vertical bars indicate the standard deviation over 20 measures.

\subsection{VECSEL setup}

The cavity geometry used is illustrated on Fig 4 and Fig 5. The folded ring cavity is formed by the VECSEL chip, the SESAM, a highly reflective mirror $\mathrm{M}_{1}$ of radius of curvature of $75 \mathrm{~mm}$, and a flat output coupler (OC) with a reflectivity of $99.2 \%$. The total cavity length is $136 \mathrm{~mm}$. The SESAM is placed at the beam waist, at a distance of $68 \mathrm{~mm}$ from the mirror $\mathrm{M}_{1}$, to provide a minimal mode size on the SESAM. The VECSEL chip is placed at $34 \mathrm{~mm}$ from the saturable absorber, a quarter of the total cavity length, to provide an equal pumping duration and gain recovery for each pulse direction. This geometrical rule is not very stringent for cavity lengths $>100 \mathrm{~mm}$, as the gain will recovers close to its full value between two consecutive pulses. The position of the flat OC is chosen to ensure $\left(\mathrm{L}_{3}+\mathrm{L}_{4}\right)=\left(\mathrm{L}_{1}+\mathrm{L}_{2}\right)=68 \mathrm{~mm}$, with an angle of incidence on the SESAM and OC of $7^{\circ}$. This geometry gives a mode waist (radius) of $152 \mu \mathrm{m}$ on the VECSEL and $85 \mu \mathrm{m}$ on the SESAM. The mode area ratio is thus only 3.2 , a factor 3 smaller than the lower limit usually adopted. ${ }^{14}$ The fiber-coupled pump diode module is focused onto the VECSEL chip at a small angle $\left(\sim 20^{\circ}\right)$, giving a beam diameter of about $280 \mu m$.

It is clear from Fig 5 that if the counter-propagating pulses are synchronized on the SESAM, the gain recovery time will be equal, providing a symmetrical amplification of the two pulses. We should note that in a CW regime, 


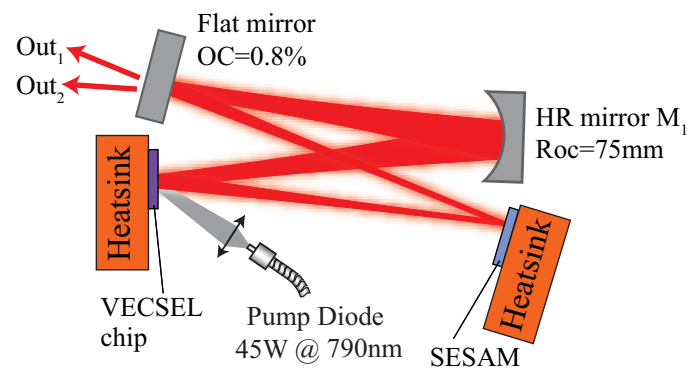

Figure 4. Schematic layout of the VECSEL device.

the interference pattern from the counter-propagating beams will be present both on the VECSEL chip and the SESAM. This means that they will saturate the absorber more easily, helping the transition from the CW to the modelocked regime. A synchronization of the pulses in the gain medium is not probable as they would better saturate the gain and would undergo a maximum absorption in the SESAM, which would not be energetically favorable.

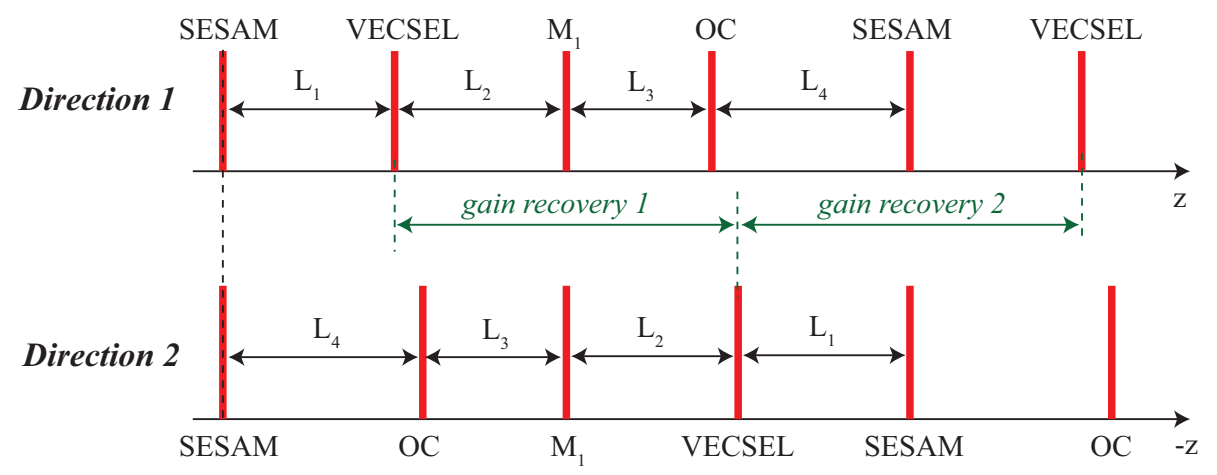

Figure 5. Relative distance of the cavity elements for each direction of propagation when the pulses are synchronized on the SESAM.

\section{MODELOCKING RESULTS}

In this section, we present the experimental results obtained with the setup described previously. For the pulses characterizations, the temperature of the VECSEL chip was kept at $5^{\circ} \mathrm{C}$ with a thermoelectric controller, and the temperature of the SESAM was varied to adjust the amount of absorption. The QW absorption band edge was close to $980 \mathrm{~nm}$ at room temperature, providing a negligible absorption and hence a maximum reflectivity at the lasing wavelength of $990 \mathrm{~nm}$, facilitating the cavity alignment. Once the cavity is aligned and lasing, the SESAM temperature is adjusted to a higher temperature, shifting the absorption spectra at a rate of $+0.3 \mathrm{~nm} / \mathrm{K}$. The VECSEL switches from CW operation to a modelocked state at a SESAM temperature of $30^{\circ} \mathrm{C}$. We recorded the output power and the pulses duration from one output beam versus the pump power and SESAM temperature (Fig. 6).

At a SESAM temperature of $35^{\circ} \mathrm{C}$, we obtained the highest average power of $300 \mathrm{~mW}$ with a pulse duration of $250 \mathrm{fs}$ at a repetition rate of $2.2 \mathrm{GHz}$, giving a peak power of $480 \mathrm{~W}$. In the following, we will call this laser state the regime 1. When the SESAM temperature is increased to $45^{\circ} \mathrm{C}$, the maximum power in a single pulse regime drops to $225 \mathrm{~mW}$ and the pulse duration get as short as $195 \mathrm{fs}$, giving a peak power of $460 \mathrm{~W}$. We will call this laser state the regime 2. For both SESAM temperatures, the pulse duration follow the same trend : the modelocking regime is unstable close to the lasing threshold, then it gets stable and the pulse duration gets shorter as the pump power is increased. This pulse shortening with a higher pump power is likely the result of the gain broadening, and of the shifting of the central wavelength closer to the center of the DBR stop-band, where the GDD is more favorable. Then, at very high pump power the higher gain and fast gain recovery enable 


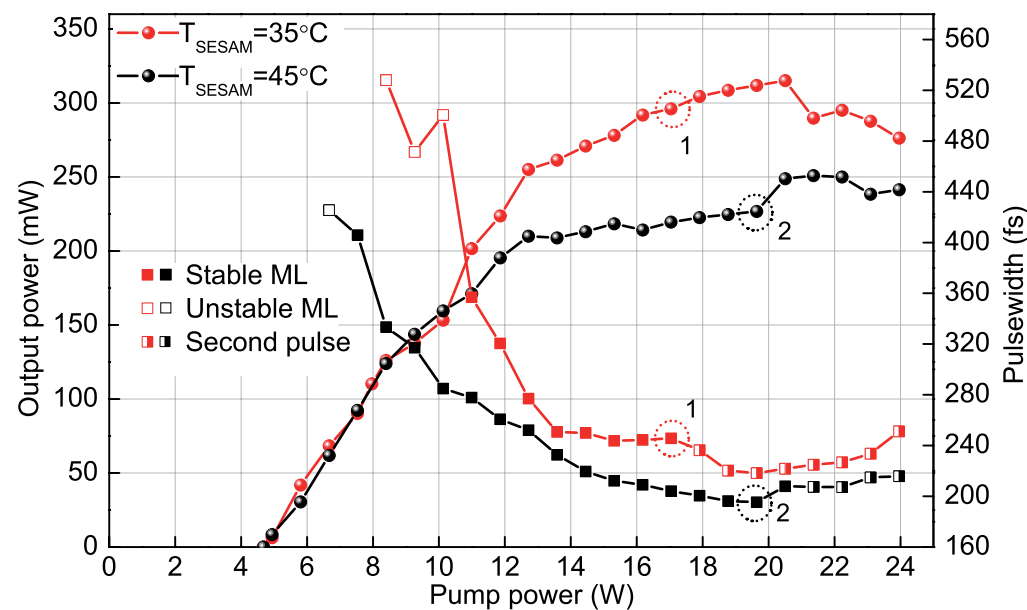

Figure 6. Output power from one output beam versus incident pump power at different SESAM temperature. The corresponding pulse durations are also plotted (right axis). The empty, full and half full squares represent respectively an unstable, a stable single pulse, and a stable with a secondary pulse modelocking regime. The two regimes circled and labeled "1" and "2" are further investigated.

a stable but smaller secondary pulse, lowering the main pulse peak power. The secondary pulse appears at higher pump power when the SESAM temperature is higher, above 17 and $21 \mathrm{~W}$ for a temperature of $35^{\circ} \mathrm{C}$ and $45^{\circ} \mathrm{C}$ respectively. This is likely due to the higher SESAM absorption at higher temperature that needs to be compensated with a higher gain to enable a secondary pulse.

The power, the spectrum and the duration of the two output beams have been measured simultaneously and are identical. The Fig. 6 also shows that a stable modelocking regime is obtained over a wide range of pump power and SESAM temperature. The remarkable robustness of the modelocking regime allows for a wide adjustment of the laser parameters to optimize the pulse duration and/or the output power. As a comparison, when the same gain element was used as a folding mirror in a more conventional V-shaped cavity, with the same SESAM and a mode area ratio of 11 , we obtained a comparable pulse duration of $220 \mathrm{fs}$ but with only $50 \mathrm{~mW}$ of output power. Moreover, the modelocking regime was observed only with a limited range of pump power, between 4.5 and $6 \mathrm{~W}$ and between 11 and $13 \mathrm{~W}$, and only with a SESAM temperature restricted to the $[45-53]^{\circ} \mathrm{C}$ range.

The two modelocked regimes were further investigated with ultrafast pulse diagnostic tools. The Fig. 7 displays the autocorrelation trace of the regime 2, recorded with a second harmonic non-collinear FemtoChrome autocorrelator. The cross correlation between the two outputs beams was also recorded with a long range non-

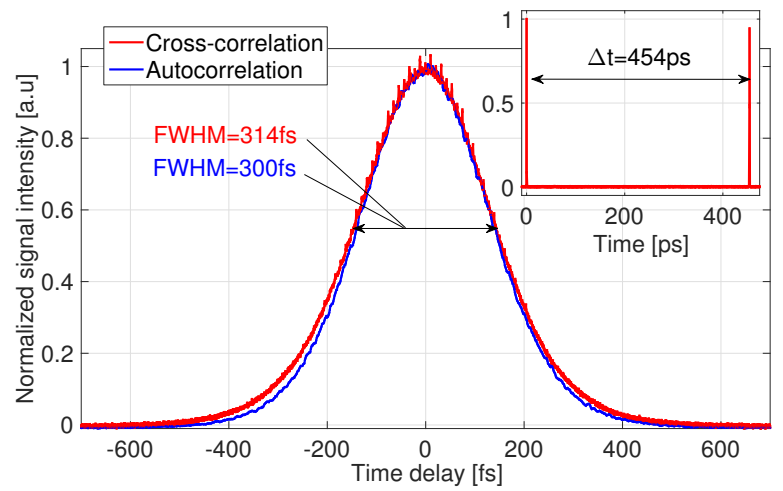

Figure 7. Measured second-harmonic-generation (SHG) non-collinear autocorrelation and cross correlation of the two counter propagative beams. In inset is the cross correlation on a long scanning range ( $i 1 F S R$ ) showing a fundamental modelocking without any side pulses. 


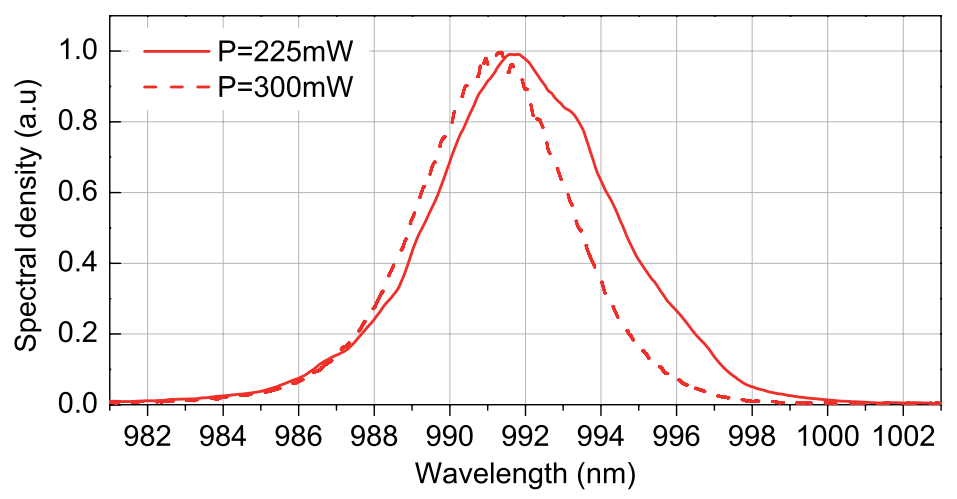

Figure 8. Optical spectrum of the two regimes investigated.

collinear home build correlator and is also plotted for comparison. The FWHM of the auto and cross correlation is respectively $300 \mathrm{fs}$ and $314 \mathrm{fs}$, with an almost perfect sech ${ }^{2}$ pulse shape, showing the synchronization of the counterpropagative pulses in the SESAM. This means that the simple combination of the two linearly polarized outputs would provide $920 \mathrm{~W}$ of peak power with a sub-200fs pulse duration. We should note that the measurement of the cross correlation of the two beams is a good indication of the jittering time of the beams since any jitter or a repetition rate difference would cause the cross correlation to be significantly longer than the autocorrelation trace. The difference of $14 \mathrm{fs}$ observed here indicates a low timing jitter, especially if we consider that the cross correlation was measured with a fairly slow scanning time $(200 \mu \mathrm{s} / \mathrm{fs})$ compared to the autocorrelation $(160 \mathrm{ps} / \mathrm{fs})$, and thus integrates more of the low frequency noise.

The optical spectra of the two regimes are shown on Fig. 8. The spectra are relatively smooth and are free of continuous wave breakthroughs, indicating a clean modelocking state. The lower SESAM temperature and lower pump power of the regime 1 gives an output spectrum centered at $991 \mathrm{~nm}$, whereas the spectrum of the regime 2 is slightly red shifted to $992 \mathrm{~nm}$, with a respective bandwidth of $4.6 \mathrm{~nm}$ and $6.8 \mathrm{~nm}$. This correspond to a time-bandwidth product of 0.35 and 0.4 indicating nearly bandwidth limited $\operatorname{sech}^{2}$ pulses $(\mathrm{x} 1.1$ and $\mathrm{x} 1.3$ the Fourier limit).

The spectral phase of the pulses was measured using a non collinear second-harmonic-generation frequency-

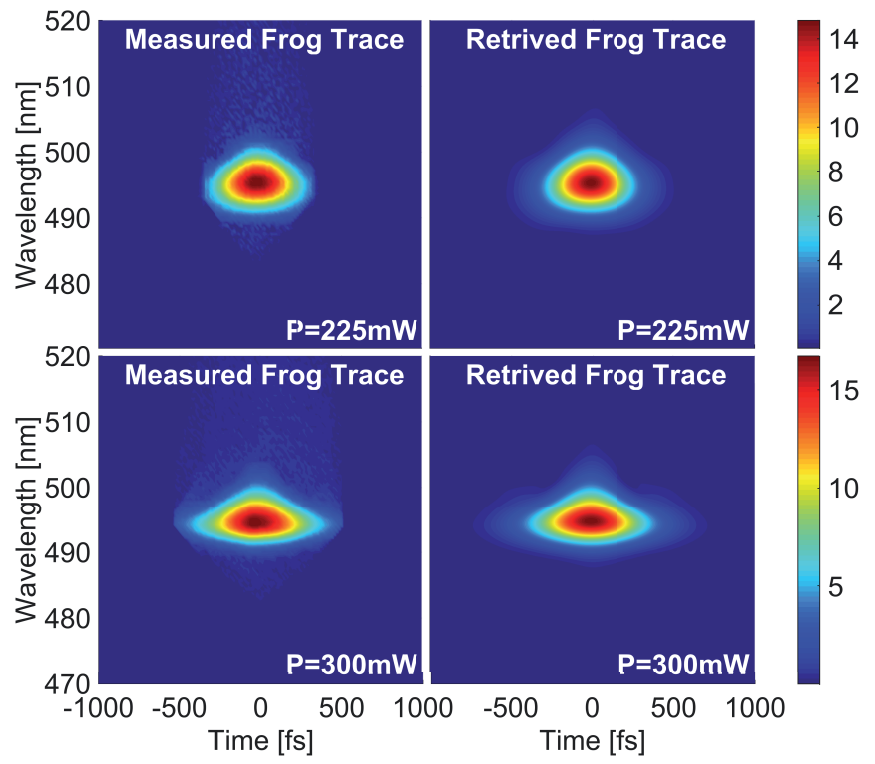

Figure 9. Measured and retrieved SHG-FROG spectrogram of the output pulses in regime 1 (bottom, error $=0.002$ ) and in regime 2 (top, error $=0.003)$. 


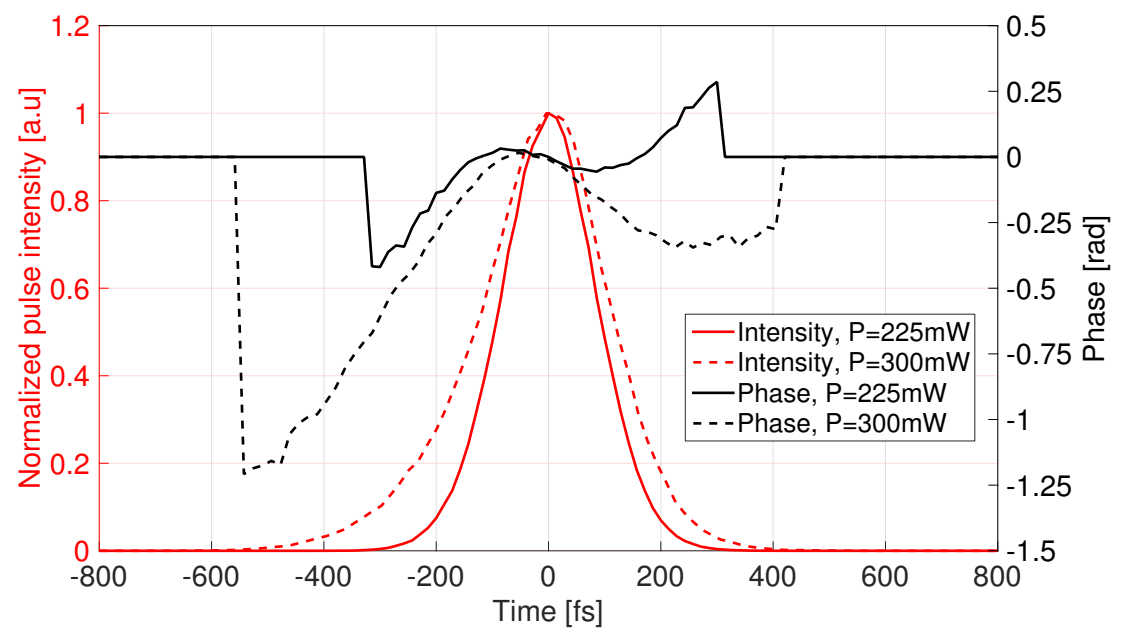

Figure 10. Intensity and phase of the pulses in the 2 regimes investigated, inferred from the SHG-FROG spectrogram.

resolved optical gating (SHG-FROG) instrument. The measured and retrieved spectrogram, reconstructed with a grid size of 256 by 256 points are shown on Fig. 9 . The very small error between the signal guess and the data constraint indicates a high degree of accuracy for the spectral phase inferred.

The pulse intensity and the phase inferred from the FROG measurement are plotted on Fig 10 for the two regimes. It shows a pulse duration of $248 \mathrm{fs}$ for the regime 1 and $194 \mathrm{fs}$ for the regime 2 , in excellent agreement with the independently measured autocorrelation. The phase spectra exhibit a non linear chirp, which could limit the pulse duration achievable.

\section{SUMMARY}

We presented a colliding pulse modelocking scheme for the generation of sub-200fs pulses from a VECSEL at high repetition rate and high average power. The significant reduction of the saturation fluence of the absorber and the increased robustness provided by this novel cavity concept was exploited to demonstrate a total output peak power of $920 \mathrm{~W}$ with a $195 \mathrm{fs}$ pulse duration. The two counter-propagating beams were characterized and were found to be identical and perfectly synchronized on the SESAM, with negligible jitter. The autocorrelation and FROG spectrogram of the pulses are in excellent agreement and exhibit pulses nearly Fourier-transform limited, demonstrating clean modelocking states. To further improve the performances and reach several $\mathrm{kW}$ of peak power at a repetition rate of several $\mathrm{GHz}$, a possible strategy could be to further increase the average power using a larger transverse mode, while maintaining a similar pulse fluence, and to further reduce the pulse duration by optimizing the GDD and third order dispersion. This task could be achieved with the use of a multilayer AR-coating and of numerically optimized structures, with a non uniform placement of the QW on the field antinodes. This peak power scaling could enable the direct generation of octave spanning coherent supercontinuum in a photonic crystal fiber from a VECSEL. The cavity geometry could also be adapted for other type of applications, for example to realize an optical gyroscope using the Sagnac effect.

\section{ACKNOWLEDGMENTS}

We thank Ganesh Balakrishnan and Sadhvikas Addamane of the University of New Mexico for providing the semiconductor saturable absorber, and Jason Jones of the University of Arizona for helpful discussions.

This material is based upon work supported by the Air Force Office of Scientific Research under award ID FA9550-14-1-0062. 


\section{REFERENCES}

[1] Laurain, A., Mart, C., Hader, J., Moloney, J., Kunert, B., and Stolz, W., "15W single frequency optically pumped semiconductor laser with sub-Mhz linewidth.," Photonics Technol. Lett. IEEE 26(2), 131-133 (2014).

[2] Klenner, A., Mayer, A. S., Johnson, A. R., Luke, K., Lamont, M. R. E., Okawachi, Y., Lipson, M., Gaeta, A. L., and Keller, U., "Gigahertz frequency comb offset stabilization based on supercontinuum generation in silicon nitride waveguides," Opt. Express 24, 11043-11053 (2016).

[3] Youg, M. D., Backus, S., Durfee, C., and Squier, J., "Multiphoton imaging with a direct-diode pumped femtosecond Ti:Sapphire laser," Journal of Microscopy 249(2), 83-86 (2013).

[4] Liu, X., Du, D., and Mourou, G., "Laser ablation and micromachining with ultrashort laser pulses," IEEE Journal of Quantum Electronics 33, 1706-1716 (1997).

[5] Hönninger, C., Paschotta, R., Morier-Genoud, F., Moser, M., and Keller, U., "Q-switching stability limits of continuous-wave passive mode locking," J. Opt. Soc. Am. B 16, 46-56 (1999).

[6] Butkus, M., Viktorov, E. A., Erneux, T., Hamilton, C. J., Maker, G., Malcolm, G. P. A., and Rafailov, E. U., "85.7 Mhz repetition rate mode-locked semiconductor disk laser: fundamental and soliton bound states," Opt. Express 21, 25526-25531 (2013).

[7] Mangold, M., Zaugg, C. A., Link, S. M., Golling, M., Tilma, B. W., and Keller, U., "Pulse repetition rate scaling from 5 to 100Ghz with a high-power semiconductor disk laser," Opt. Express 22, 6099-6107 (2014).

[8] Bek, R., Kahle, H., Schwarzbäck, T., Jetter, M., and Michler, P., "Mode-locked red-emitting semiconductor disk laser with sub-250 fs pulses," Applied Physics Letters 103(24) (2013).

[9] Härkönen, A., Grebing, C., Paajaste, J., Koskinen, R., Alanko, J. P., Suomalainen, S., Steinmeyer, G., and Guina, M., "Modelocked GaSb disk laser producing 384fs pulses at $2 \mu \mathrm{m}$ wavelength," Electron. Lett 47, 454 -456 (2011).

[10] Waldburger, D., Link, S. M., Mangold, M., Alfieri, C. G. E., Gini, E., Golling, M., Tilma, B. W., and Keller, U., "High-power 100fs semiconductor disk lasers," Optica 3, 844-852 (2016).

[11] Wilcox, K. G., Tropper, A. C., Beere, H. E., Ritchie, D. A., Kunert, B., Heinen, B., and Stolz, W., "4.35kW peak power femtosecond pulse mode-locked VECSEL for supercontinuum generation," Opt. Express 21, 1599-1605 (2013).

[12] Kilen, I., Hader, J., Moloney, J. V., and Koch, S. W., "Ultrafast nonequilibrium carrier dynamics in semiconductor laser mode locking," Optica 1, 192-197 (2014).

[13] Laurain, A., Marah, D., Rockmore, R., McInerney, J., Hader, J., Perez, A. R., Stolz, W., and Moloney, J. V., "Colliding pulse mode locking of vertical-external-cavity surface-emitting laser," Optica 3, 781-784 (Jul 2016).

[14] Lorenser, D., Unold, H., Maas, D., Aschwanden, A., Grange, R., Paschotta, R., Ebling, D., Gini, E., and Keller, U., "Towards wafer-scale integration of high repetition rate passively mode-locked surface-emitting semiconductor lasers," Applied Physics B 79(8), 927-932 (2004). 